\title{
Structuring an Ontology for Mathematical Modelling Wastewater Flow through Porous Media
}

\author{
Stephanos D. V. Giakoumatos \\ School of Maritime and Industrial Studies, University of Piraeus, Piraeus, Greece \\ Email: sgiakou@hotmail.com,sgiakoum@unipi.gr
}

How to cite this paper: Giakoumatos, S.D.V. (2021) Structuring an Ontology for Mathematical Modelling Wastewater Flow through Porous Media. Intelligent Information Management, 13, 156-167. https://doi.org/10.4236/iim.2021.132008

Received: February 1, 2021

Accepted: March 23, 2021

Published: March 26, 2021

Copyright ( 2021 by author(s) and Scientific Research Publishing Inc. This work is licensed under the Creative Commons Attribution International License (CC BY 4.0).

http://creativecommons.org/licenses/by/4.0/

\section{(c) (i) Open Access}

\begin{abstract}
Single or multi-layer infiltration rate models describe water penetration into soil porous zone at a variety of comparable complexity levels. Commonly, those models are indicating a route leading from general to more specific case depending on the mathematical structure and the simulated ongoing phenomenon. For the purpose of wastewater flow quantification through porous media, an algorithmic procedure was developed which includes certain interconnected activity stages and decision nodes. Furthermore, a schematic cross shaped representation of the infiltration rate models' ontology is presented within a route, leading from general to more specific models and evaluation criteria are introduced to determine highest ranking and thus the best model amid those placed on the horizontal axis of the cross shaped scheme, via a simple Multi-Criteria Analysis Decision Making.
\end{abstract}

\section{Keywords}

Ontology, Infiltration Models, Porous Media, VPCs, Multi-Criteria Analysis Decision Making

\section{Introduction}

This Complexity is the main characteristic of mathematical models that simulate wastewater flow through porous media either under laboratory/industrial conditions or in the Nature, where soil pollution takes place. By increasing complexity, we can 1) improve the explainability of the dependent variable, and 2) achieve parameters identification at deeper phenomenological levels; on the other hand, applicability difficulties and information acquisition/processing/ storing/retrieval cost also increase. Since such issues imply a tradeoff, we can 
determine the optimal value of a complexity index, including the number of independent Variants Parameters, Coefficients (VPCs) and the phenomenological knowledge depth, representing numerical and structural complexity, respectively.

Numerous models are not independent to each other but interconnected as "members" of the same category or "rings" in the same chain, representing a derivation path. Given in such a mode they build up an Ontology structure in terms of modern Informatics and Logic/Methodology of Science.

Ontologies entails concepts suitably bound by well-defined relationships in specific scientific fields which are applied in artificial intelligence in order to provide to all users an interaction framework with numerous application systems i.e. communication models between (KB) users and machines [1]. Such structures appear to form an entity with concepts, relations and controlled vocabulary, while logical operators and taxonomy/partonomy functions are used for processing the relevant knowledge.

Modeling application methodology can be improved by utilizing ontological structures for all stages of model building. This paper explores mechanisms by setting up algorithmic procedure as the path to build up an Ontology of mathematical models to approach wastewater flow quantification through porous media. Thus, it is manageable ontological entities to be incorporated into Knowledge Bases (KBs) in knowledge engineering over infiltration modelling discipline that combined with artificial intelligence and expert systems, information can be easily retrieved and applied in a variety of applications [2] [3] [4].

\section{Methodology}

For structuring an Ontology of mathematical models to quantify wastewater flow through porous media an algorithmic procedure was designed/developed which includes the following 30 activity stages and 8 decision nodes while the interconnection is shown in Figure 1 [5] [6] [7].

1. Characterization of the waste water to be examined as regards its flow.

2. Description of the porous system under consideration.

3. Collection of the relevant VPCs.

4. Selection-categorization of the m most relevant mathematical models (including new ones synthesized adhoc) based on the aforementioned VPCs for quantifying this process (taxonomy function within the ontology under design).

5. Checking for their dimensional homogeneity

6. Investigation of the mathematical derivation paths where each model belongs to (upwards partial partonomy function within the ontology under design).

7. Mathematical model restoration by eliminating the error.

8. Identification of the mathematical derivation paths where each model belongs to (upwards-downwards total partonomy function). 
9. Determination of $\mathrm{n}$ criteria to be used for choosing the best mathematical model from the set of alternatives selected in stage 4 .

10. Assignment of grades onto the elements of the multi-criteria $n \times m$ preference matrix (by using experts' opinion) in fuzzy version to count for uncertainty.

11. Performance of multi-criteria analysis.

12. Experimental validation of the proposed mathematical model.

13. Scale-up to the required level and estimation of the tolerance limits expected to constrain applicability.

14. Sensitivity-robustness analysis by changing the values assigned to the elements of the criteria vector and the preference matrix.

15. Selection of the minimum number $\mathrm{p}$ of VPCs required to describe the phenomenon under examination.

16. Merging in accordance with the respecting physical knowledge.

17. Consideration of the $r$ primary dimensions or fundamental quantities common to all VPCs and the construction of a vector space with $r$ linear independent vectors, where each of the remaining $(n-r)$ vectors can be expressed as a linear combination of the $r$ independent ones, according to the corresponding techniques of Linear Algebra and Group Theory.

18. At least one primary dimension splitting into g sub-dimension, thus increasing by ( $g$ - 1) r number and increasing equally the number of dimensionless groups obtained through the dimensional matrix.

19. Confirmation of the dimensional matrix rank by determining the number of non-zero rows which are linearly independent) in the echelon form of the dimensional matrix according to the usual linear algebra technique.

20. Determination at the complete set of dimensionless groups according to Rayleigh's method of indices and the Buckingham's pi theorem, keeping the rules on independence of VPCs, as provided by Linear Algebra.

21. Rearrangement of VPCs in this set to obtain an equivalent complete set with the maximum number of recognizable dimensionless groups, i.e. groups with physical meaning, usually expressed as ratios properly formed to facilitate scale-up/down.

22. Experimental design and execution of measurements.

23. Estimation of parameter's values of the linearized model.

24. Fine tuning of this estimation by using non-linear least squares.

25. Multi-criteria choice of the best model.

26. Comparison with similar models/groups from literature.

27. Ontology of dimensionless groups.

28. Structure of the mathematical models' ontology

29. Development/operation/updating of an internal Knowledge Base (KB)

30. Searching in external KBs by means of an Intelligent Agent to enrich the internal one. 


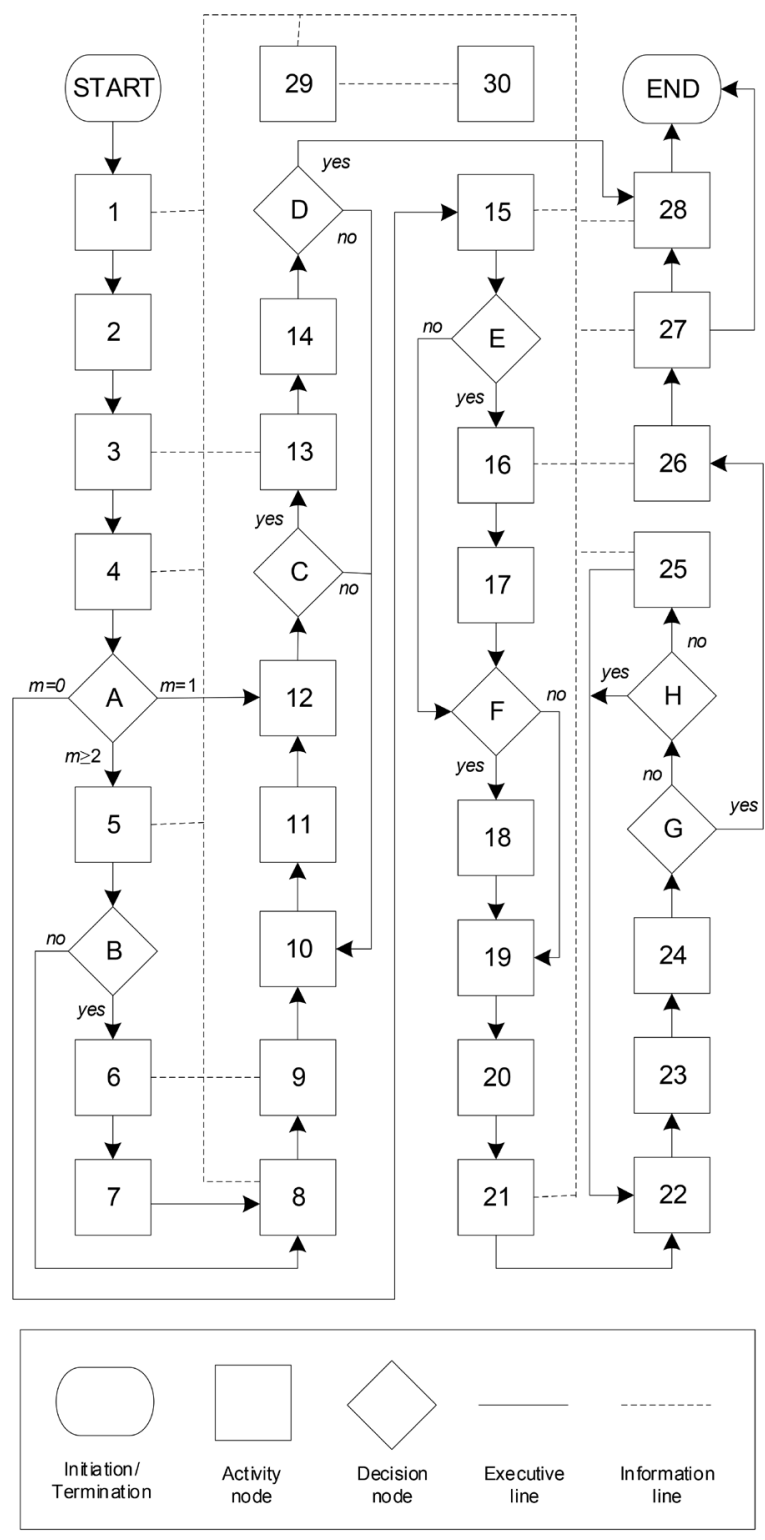

Figure 1. The algorithmic procedure developed for structuring an Ontology of mathematical models to quantify wastewater flow through porous media.

A. Is $m \geq 2$ or $m=1$ or $m=0$ ?

B. Is at least one of them dimensionally inhomogeneous?

C. Is it valid to preset confidence intervals at the required significance level?

D. Are the numerical results of sensitivity - robustness analysis within the range corresponding to the tolerance limits?

E. Is it considered to be necessary to minimize $p$ further without losing the initial information (i.e. decreasing the information granularity level) required to describe adequately this phenomenon?

F. Is splitting of at least one primary dimension indispensable in order to enhance explainability-predictability?

G. Is SEE within the pre-determined range? 
$\mathrm{H}$. Is there at least one complete set remained unexamined so far?

Considering e.g. water penetration into soil porous zone, numerous single or multi-layer infiltration rate models can be employed to approach water infiltration development at comparable complexity levels.

Infiltration rate models are indicating a route leading from general to more specific ones according to the mathematical structure and simulated operation. Hereunder, certain infiltration models are presented which simulate infiltration phenomena through porous media.

Green-Ampt (1911) (3.1 in Figure 2)

Simple model for one-dimensional vertical infiltration through ponded homogeneous soil of uniform antecedent moisture content. The water flow in the saturation zone was caused by constant soil water suction at the wetting front and gravity of soil water. Considering that the depth of the ponding is negligible [8]:

$$
f(t)=K_{S} *\left[1+\left(\frac{H_{o}+h_{s}}{Z_{f}}\right)\right]
$$

where $K_{S}$ is considered to be the saturated hydraulic conductivity, [LT']

$f$ is the infiltration rate, $\left[\mathrm{LT}^{-1}\right]$ or infiltration capacity $\Delta \theta$ is the moisture content change $\left[\mathrm{L}^{3} \cdot \mathrm{L}^{-3}\right]$,

$\theta_{s}$ and $\theta_{i}$ are the saturated and initial (volumetric) soil water contents respectively,

$Z_{f}$ is the wetting front depth [L]

$H_{o}$ is the depth of ponding water [L]

$h_{\mathrm{s}}$ is the capillary suction head at the wetting front $[\mathrm{L}]$

t: time

3.1

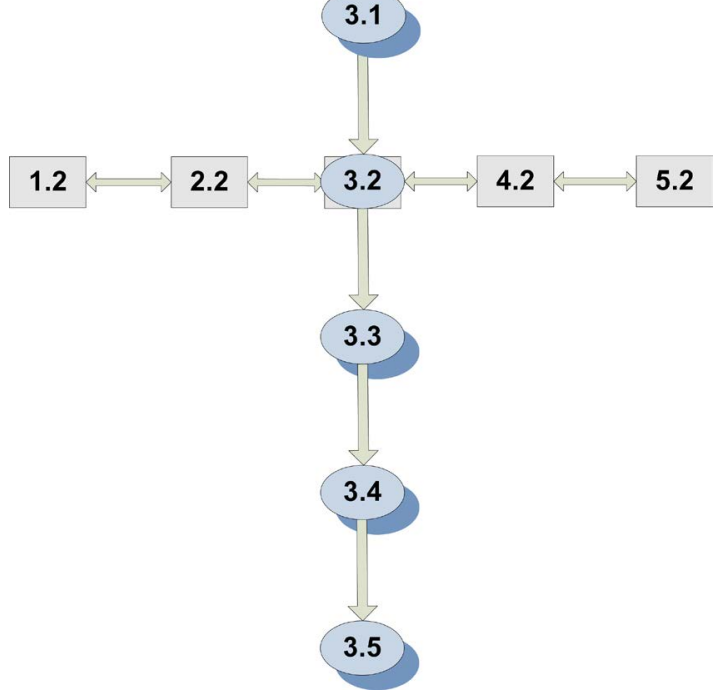

Figure 2. Schematic representation of the infiltration rate models ontology. The horizontal axis presents "is a" relations, defining a set, the members of which can be used as performance alternatives at comparable complexity levels. The vertical axis presents "part of" relations within a route leading from general to more specific ones. 


\section{Chu \& Mariño (2005) (3.2 in Figure 2)}

Modified Green-Ampt model for computing infiltration into $n$ layered soils at initially ponded conditions \& non-uniform soils. When the wetting front is in layer $n$ at location $Z\left(Z_{n-1}<Z<Z_{n}\right)$, the infiltration rate and cumulative infiltration (Figure 3 ) can be expressed as [9]:

$$
f_{Z}=\frac{Z+h_{s n}}{\sum_{j=1}^{n-1} \frac{Z_{j}-Z_{j-1}}{K_{j}}+\frac{Z-Z_{n-1}}{K_{n}}}
$$

$Z$, wetting front depth location [L]

$Z_{j}$ location of the $j$ layer

$K_{j} K_{n}$ is the effective hydraulic conductivity of layer $j, n$, respectively [L/T]

$f_{z}$ is the infiltration rate at the location $Z,\left[\mathrm{LT}^{-1}\right]$

$H_{Z}$ is the hydraulic head at $Z$ wetting front, [L]

$h_{s n}$ is the pressure head (suction) in layer $n,[\mathrm{~L}]$

Schmid (1990) (3.3 in Figure 2)

Model for time dependent infiltration rate and cumulative infiltration based on Mein \& Larson model (1973) [10]:

$$
\begin{gathered}
f(t)=I_{i}+\left(I_{p}-I_{i}\right)\left[1+2 \frac{\left(I_{p}-K_{s}\right)^{2}}{K_{s}\left(H_{o}+h_{s}\right) \Delta \theta} t^{*}\right]^{-\frac{1}{2}} \\
0<t^{*}=t-t_{p}
\end{gathered}
$$

where:

$f$ is the infiltration rate, $\left[\mathrm{LT}^{-1}\right]$

$I(t)$ is the rainfall intensity, [L]

$I_{i}$ is the antecedent rainfall intensity, [L]

$K_{S}$ is the saturated hydraulic conductivity, $\left[\mathrm{LT}^{-1}\right]$

$I_{p}$ the rainfall intensity at the ponding time $t_{p}$, [L]

$\Delta \theta$ is the moisture content change $\left[\mathrm{L}^{3} \cdot \mathrm{L}^{-3}\right]$,

$h_{s}$, the capillary suction head at the wetting front $[\mathrm{L}]$

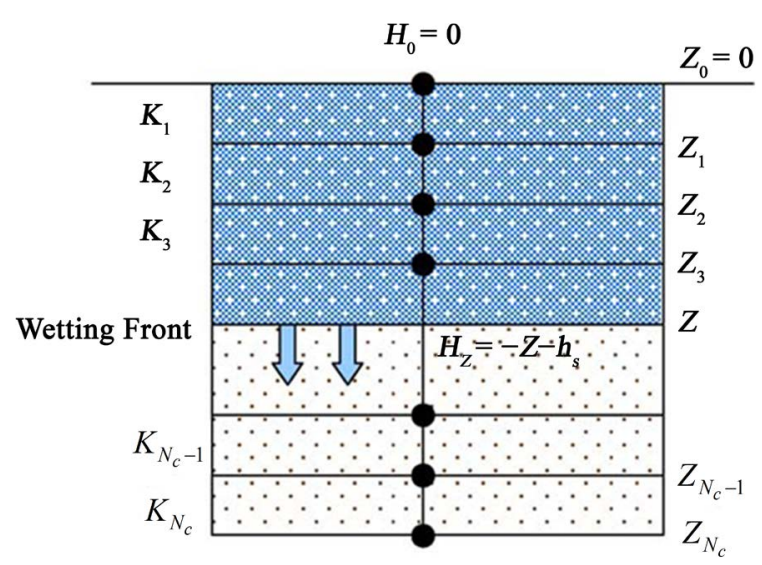

Figure 3. Infiltration into a layered soil profile under an initially ponded condition [5]. 
$H_{o}$, depth of ponding water over the soil surface [L]

t. time

Mein and Larson (1973) (3.4 in Figure 2)

Simple 2-stage model for infiltration prediction before and after surface ponding initial Uniform moisture content assumption under rainfall with a constant intensity [11].

Case 1: infiltration prior to runoff and the time to the beginning of runoff, with $I \geq K_{s}$

Case 2: infiltration after runoff begins

$$
f_{p}=K_{s} *\left[1+\left(\frac{\Delta \theta * h_{a v}}{F}\right)\right]
$$

$f_{p}$ is the infiltration capacity, $\left[\mathrm{LT}^{-1}\right]$

$K_{s}$ is the saturated conductivity, [LT $\left.{ }^{-1}\right]$

$h_{a v}$ is the average capillary suction at the wetting front, [L]

$\Delta \theta$ is the initial moisture deficit $\left(\theta_{s}-\theta_{i}\right)$ (volume/volume), $\left[\mathrm{L}^{3} \cdot \mathrm{L}^{-3}\right]$

$F$ is the cumulative infiltration at any time, $[\mathrm{L}]$ with:

$$
h_{a v}=\int_{0}^{1} h \mathrm{~d} k_{r}
$$

where:

$$
k_{r}=\frac{K}{K_{s}}
$$

$K$ is the capillary conductivity $\theta$ dependent, $\left[\mathrm{LT}^{-1}\right]$

$k_{r}$ is the relative conductivity (dimensionless)

Salvucci \& Entekhabi (1994) (3.5 in Figure 2)

Model applicable for homogeneous soils, uniform antecedent water content, non-zero or constant ponding depth [12].

$$
\begin{gathered}
\frac{f(t)}{K_{s}}=\frac{\sqrt{2}}{2} * \tau^{-\frac{1}{2}}+\frac{2}{3}-\frac{\sqrt{2}}{6} * \tau^{\frac{1}{2}}+\frac{1-\sqrt{2}}{3} * r \\
\tau=\frac{t}{t+\chi} \\
\chi=\frac{\left(H_{o}-h_{s}\right) * \Delta \theta}{K_{s}}
\end{gathered}
$$

$K_{s}$ saturated hydraulic conductivity, $\left[\mathrm{LT}^{-1}\right]$

$f$, is the infiltration rate, $\left[\mathrm{LT}^{-1}\right]$

$\Delta \theta$, is the moisture content change $\left[\mathrm{L}^{3} \cdot \mathrm{L}^{-3}\right]$,

$H_{o}$, is the depth of ponding water [L]

$h_{s}$ the capillary suction head at the wetting front [L]

$t$ is the time $[\mathrm{T}]$

Infiltration models applied onto layered soils during rainfall

Childs and Bybordi (1969) (1.2 in Figure 2) 
Green-Ampt model extension, infiltration process into layered soils with a decreasing hydraulic conductivity from the soil surface moving downwards. Constant ponding depth. Infiltration rate estimation at which the wetting front reaches some location in the $n$th layer [13].

$$
f=\frac{K_{n}\left(L+H_{o}+h_{s n}\right)}{L+\sum_{j=0}^{n} Z_{j} *\left(\frac{K_{n}}{K_{j}}-1\right)}
$$

$f$, is the infiltration rate, $\left[\mathrm{LT}^{-1}\right]$

$L$, is the depth of wetting front; [L]

$K_{n}$, saturated hydraulic conductivity of $n$th soil layer, [LT $\left.{ }^{-1}\right]$

$K_{j}$, saturated hydraulic conductivity of $j$ th soil layer, $\left[\mathrm{LT}^{-1}\right]$

$H_{o}$, depth of ponding water over the soil surface [L]

$h_{s n}$, is the capillary pressure head at the $n$ th, [L]

$n$, total number of soil layers

$Z_{j}$ depth of $j$ th layer [L]

$t$, time at which the wetting front is observed in $n$th layer, [T]

$t_{n-1}$, time at which the wetting front reaches the boundary between $(n-1)$ th layer and $n$th layer, [T]

Sonu (1986) (2.2 in Figure 2)

Model in heterogeneous, multi-layered soils, includes the effects of viscosity of fluid on the infiltration rate [14].

$$
\begin{gathered}
f=\frac{K_{n} *\left(L+h_{o}+h_{s n}\right)}{\beta_{n} *\left(L-\sum_{j=1}^{n-1} z_{j}\right)+\sum_{j=1}^{n-1}\left(\frac{K_{n}}{K_{j}}\right) * z_{j}} \\
\beta_{n}=\left(\theta_{s n}-\theta_{0 n}\right) * \int_{\theta_{0 n}}^{\theta_{s n}} \frac{-f_{w n}^{\prime \prime \prime}}{\mu_{w} * V_{r n}} \mathrm{~d} \theta
\end{gathered}
$$

where:

$n$, total number of soil layers

$h_{s n}$, capillary pressure head at the $n$th layer, [L]

$z_{j}$ depth of th layer, [L]

$K_{n}$, saturated hydraulic conductivity of $n$th layer, $\left[\mathrm{LT}^{-1}\right]$

$K_{j}$, saturated hydraulic conductivity of $j$ th layer, $\left[\mathrm{LT}^{-1}\right]$

$\theta_{s n}$, saturated water content of $n$th layer, $\left[\mathrm{L}^{3} \cdot \mathrm{L}^{-3}\right]$

$\theta_{0 n}$, initial water content of $n$th layer, $\left[\mathrm{L}^{3} \cdot \mathrm{L}^{-3}\right]$

$\beta_{n}$, viscous correction resistance, the value which need not to be evaluated at every layer of the soil

$f_{w n}$, fractional flow function evaluated for the $n$th layer, $\left[\mathrm{LT}^{-1}\right]$,

$V_{r n}$, relative viscous resistance, (dimensionless)

$\mu_{w}$, dynamic viscosity of air or water $\left[\mathrm{ML}^{-1} \cdot \mathrm{T}^{-1}\right]$

Jia and Tamai (1997) (4.2 in Figure 2)

They presented a generalized Green-Ampt model to simulate the infiltration 
into a multi-layered soil during unsteady rain. When surface ponding is occurring from the beginning of a rain event and is continuing, while the wetting front penetrates the $m$ th soil layer [15]:

$$
\begin{gathered}
f=K_{m}\left(1+\frac{A_{m-1}}{B_{m-1}+F}\right) \\
A_{m-1}=\left(\sum_{i}^{m-1} L_{i}-\sum_{i}^{m-1} \frac{L_{i} K_{m}}{K_{i}}+h_{s m}\right) \Delta \theta_{m} \\
B_{m-1}=\left(\sum_{i}^{m-1} \frac{L_{i} K_{m}}{K_{i}}\right) \Delta \theta_{m}-\sum_{i}^{m-1} L_{i} \Delta \theta_{i}
\end{gathered}
$$

where:

$F$ is the cumulative infiltration, $[\mathrm{L}]$

$f$ is the infiltration rate, $\left[\mathrm{LT}^{-1}\right]$

$t_{m-1}$ time when the wetting front reached the interface of $m$ th and $(m-1)$ th soil layers [T]

$L$ is the depth of wetting front; [L]

$L_{i}$ is the thickness of $i$ th soil layer, [L]

$K_{i}, K_{m}$ considered to be the initial hydraulic conductivity and $m$ th soil layer respectively, $\left[\mathrm{LT}^{-1}\right]$

$\Delta \theta_{m}, \Delta \theta_{i}$ the change in moisture content ( $m$ layer \& initial layer), [ $\left.\mathrm{L}^{3} \cdot \mathrm{L}^{-3}\right]$,

$h_{s m}$ is the pressure head (suction) in layer $m$, [L]

Han et al., (2001) (5.2 in Figure 2)

Extended form of the original Green-Ampt model for infiltration into layered, non-uniform soils [16].

$$
\begin{gathered}
f=\bar{K}_{s} \frac{Z_{f}+H_{o}+h_{f}}{Z_{f}} \\
\overline{K_{s}}=\frac{\sum_{j=1}^{M+1} D_{j}}{\sum_{j=1}^{M+1} \frac{D_{j}}{K_{s, j}}}
\end{gathered}
$$

$\overline{K_{s}}$ consider to be the average saturated hydraulic conductivity of soil layers, $\left[\mathrm{LT}^{-1}\right]$

$f$ is the infiltration rate, $\left[\mathrm{LT}^{-1}\right]$ or infiltration capacity $Z_{f}$ is the wetting front depth $[\mathrm{L}]$

$H_{o}$ is the depth of ponding water [L]

$D_{j}$ is the thickness of soil layer number $j[\mathrm{~L}]$

$M$ subscript denotes the saturated layer number

$K_{s, j}$ denotes the saturated hydraulic conductivity of soil layer $j$

$h_{f}$ is the wetting front suction head, [L]

The infiltration models depicted on horizontal axis of schematic representation in Figure 2 shall undergo evaluation by means of a simplified Multi-Criteria Decision Making (MCDM) methodology [3]. Six criteria determine the final 
score accumulation and thereof the final ranking (see Table 1). The final ranking of the available infiltration models leads to the optimal final selection as the $25^{\text {th }}$ activity stage described in Chapter 2 . Each criterion has its own gravity (weight) (see $2^{\text {nd }}$ column of Table 2). Criterion of higher weight value entails more influential impact on the final ranking. The overall value estimation is in fact the best model performance in a tradeoff evaluation. During the (MCDM) procedure an evaluation of each model over a criterion is carried out and a grade within 0 - 10 climax was given. The higher the grade the better conformity of the model to the criterion direction. The selected criteria are given below in Table 1 .

The scores presented in Table 2 form a $6 \times 5$ evaluation matrix $\left(\boldsymbol{J}_{k \times l}\right)$.

$$
\boldsymbol{J}=\left(\begin{array}{ccc}
a_{1,1} & \cdots & a_{5,1} \\
\vdots & \ddots & \vdots \\
a_{6,1} & \cdots & a_{6,5}
\end{array}\right)
$$

where:

$$
\begin{aligned}
& k=1,2, \cdots, 6 \\
& l=1,2, \cdots, 5
\end{aligned}
$$

$a_{k, l}$ represents the score achieved each model to fulfill the corresponding criterion and is acquired by author's empirical knowledge after scrutinizing all relative publications. The values of $\boldsymbol{J}$ are already normalized since the evaluation range was set out to be between $0-10$.

Table 1. Multi-criteria evaluation score for the horizontal axis infiltration models.

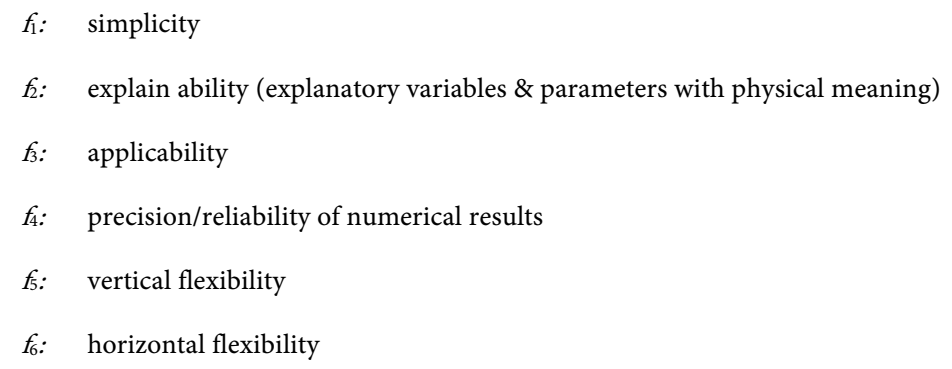

Table 2. Multi-criteria evaluation score for the horizontal axis infiltration models.

\begin{tabular}{ccccccc}
\hline \multirow{2}{*}{ factor } & weight & \multicolumn{5}{c}{ model } \\
\cline { 3 - 6 } & 10.0 & 7.1 & 2.3 & 3.2 & 4.2 & 5.2 \\
\hline$f_{1}$ & 20.9 & 3.0 & 6.0 & 3.1 & 5.2 & 3.2 \\
$f_{2}$ & 23.5 & 6.0 & 2.3 & 6.5 & 4.5 & 4.5 \\
$f_{3}$ & 18.1 & 4.5 & 4.7 & 4.9 & 4.3 & 4.0 \\
$f_{4}$ & 15.5 & 3.0 & 3.1 & 4.2 & 3.6 & 3.4 \\
$f_{5}$ & 12.0 & 6.0 & 7.1 & 5.4 & 5.3 & 5.5 \\
$f_{6}$ & 100.0 & 474.7 & 420.8 & 541.1 & 471.7 & 440.7 \\
$S W S_{i}$ & & & & & & \\
\hline
\end{tabular}


The second column of Table 2 represents the column vector $f$ of the impact factor of each criterion. Thus:

$$
\boldsymbol{f}=\left[\begin{array}{l}
f_{1} \\
f_{2} \\
f_{3} \\
f_{4} \\
f_{5} \\
f_{6}
\end{array}\right]
$$

$\left(S W S_{i}\right)$ : stands for "Sum of Weighted Score" for each of the six models to be evaluated. Therefore:

$$
S W S_{i}=\boldsymbol{J} * \boldsymbol{f}=\left(\begin{array}{ccc}
a_{1,1} & \cdots & a_{5,1} \\
\vdots & \ddots & \vdots \\
a_{6,1} & \cdots & a_{6,5}
\end{array}\right) *\left[\begin{array}{c}
f_{1} \\
f_{2} \\
f_{3} \\
f_{4} \\
f_{5} \\
f_{6}
\end{array}\right]
$$

The higher the $S W S_{i},(i=1,2, \cdots, 6)$, the better the overall performance of the model regarding the above given criteria.

\section{Conclusion}

The algorithmic procedure developed for structuring an Ontology of mathematical models presented analytically in Chapter 2, enables programmers to approach the physical infiltration phenomenon by quantifying wastewater flow through porous media. The final multi-criteria analysis merely demonstrates a simple way to perform a ranking by using preselected model criteria. In our case the evaluation results presented are as follow i.e. $3.2>1.2>4.2>5.2>2.2$, where the symbol ">" stands for "better than". Thereof, the modified Green-Ampt model (3.2) for computing infiltration into $n$-layered soils at initially ponded conditions \& non-uniform soils gives the optimum approach in terms of predetermined criteria $f_{1}-f_{6}$. The second best is Childs and Bybordi model, an extension of the classic GreenAmpt model, simulating the infiltration process into layered soils with a decreasing hydraulic conductivity from the soil surface downwards.

\section{Conflicts of Interest}

The author declares no conflicts of interest regarding the publication of this paper.

\section{References}

[1] Weng, S.S. and Chang, H.L. (2008) Using Ontology Network Analysis for Research Document Recommendation. Expert Systems with Applications, 34, 1857-1869. https://doi.org/10.1016/j.eswa.2007.02.023

[2] Kwon, H.Y., Grunwald, S., Beck, H.W., Jung, Y., Daroub, S.H., Lang, T.A., et al. (2010) Ontology-Based Simulation of Water Flow in Organic Soils Applied to Flor- 
ida Sugarcane. Agricultural Water Management, 97, 112-122. https://doi.org/10.1016/j.agwat.2009.08.019

[3] Giakoumatos, S.D.V. and Gkionakis, A.K.T. (2021) Development of an Ontology-Based Knowledge Network by Interconnecting Soil/Water Concepts/Properties, Derived from Standards Methods and Published Scientific References Outlining Infiltration/Percolation Process of Contaminated Water. Journal of Geoscience and Environment Protection, 9, 25-52. https://doi.org/10.4236/gep.2021.91003

[4] Du, H., Dimitrova, V., Magee, D., Stirling, R., Curioni, G., Reeves, H., Clarke, B. and Cohn, A. (2016) An Ontology of Soil Properties and Processes. Proceedings of 2016 International Semantic Web Conference, Kobe, 17-21 October 2016, 30-37. https://doi.org/10.1007/978-3-319-46547-0 4

[5] Batzias, F. and Giakoumatos, S. (2016) Fuzzy Multi-Objective Ranking of Scenarios/ Technologies aiming at Reduction of $\mathrm{SO}_{\mathrm{x}}$ Emissions by Ships. International Journal of Environmental Science, 1, 309-316. https://doi.org/10.1016/S1570-7946(02)80166-3

[6] Batzias, F.A. and Marcoulaki, E.C. (2002) Restructuring the Keywords Interface to Enhance CAPE Knowledge via an Intelligent Agent. Computer Aided Chemical Engineering, 10, 829-834. https://doi.org/10.1016/S1570-7946(02)80166-3

[7] Bukhsh, Z.A., Oslakovic, I.S., Klanker, G., Hoj, N.P., Imam, B. and Xenidis, Y. (2017) Multi Criteria Decision Making: AHP Method Applied for Network Bridge Prioritization. Proceedings of the Joint COST TU1402-COST TU1406-IABSE WC1 Workshop, Zagreb, 2-3rd March 2017, 1-9. https://doi.org/10.5592/CO/BSHM2017.3.2

[8] Green, W.H. and Ampt, G.A. (1911) Studies in Soil Physics, Part 1, the Flow of Air and Water through Soils. The Journal of Agriculture Science, 4, 11-24. https://doi.org/10.1017/S0021859600001441

[9] Chu, X. and Mariño, M.A. (2005) Determination of Ponding Condition and Infiltration into Layered Soils under Unsteady Rainfall. Journal of Hydrology, 313, 195 207. https://doi.org/10.1016/j.jhydrol.2005.03.002

[10] Schmid, B.H. (1990) Derivation of an Explicit Equation for Infiltration on the Basis of the Mein-Larson Model. Journal of Hydrological Sciences, 35, 197-208. https://doi.org/10.1080/02626669009492418

[11] Mein, R.G. and Larson, C.L. (1973) Modeling Infiltration during a Steady Rain. Water Resources Research, 9, 384-394. https://doi.org/10.1029/WR009i002p00384

[12] Salvucci, G.D. and Entekhabi, D. (1994) Explicit Expressions for Green-Ampt (Delta Function Diffusivity) Infiltration and Cumulative Storage. Water Resources Research, 30, 2661-2663. https://doi.org/10.1029/94WR01494

[13] Childs, E.C. and Bybordi, M. (1969) The Vertical Movement of Water in a Stratified Porous Material: 1. Infiltration. Water Resources Research, 5, 446-459. https://doi.org/10.1029/WR005i002p00446

[14] Sonu, J. (1986) Vertical Infiltration into Stratified Soil for Groundwater Accretion. Conjunctive Water Use. Proceedings of the Budapest Symposium, Budapest, July 1986, IAHS Publishing No. 156, 365-374.

[15] Jia, Y. and Tamai N. (1997) Modeling Infiltration into a Multi-Layered Soil during and Unsteady Rain. Journal of Hydroscience and Hydraulic, 41, 31-36. https://doi.org/10.2208/prohe.41.31

[16] Han, Y., Luo, Y., Yu, Q. and Zhang, H. (2001) The Green-Ampt Model for Heterogeneous Soil Profiles. Chinese Journal of Eco-Agriculture, 9, 31-37. 